EDITORIAL

\title{
Are drug eluting stents really worth the money?
}

M Thomas

Heart 2006;92:5-7. doi: 10.1136/hrt.2005.066316

The cost effectiveness of drug eluting stents is being called into question. But is this fair in the light of all the available clinical data?

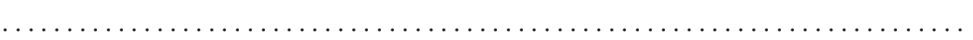

r

$\mathrm{n}$ this issue of Heart Bagust and colleagues ${ }^{1}$ report on their view of the cost effectiveness of the drug eluting stents (DES) in the UK. The remarkable conclusion of this manuscript appears to be that DES should only be used in $4 \%$ of patients on economic grounds. Previous National Institute for Health and Clinical Excellence (NICE) guidelines ${ }^{2}$ supported DES use in small vessels $(<3 \mathrm{~mm})$ and longer lesions $(>15 \mathrm{~mm})$ and it was anticipated this would result in a $30 \%$ use per lesion. The conclusions of the current paper are highly controversial and deserve further comment.

\section{SINGLE CENTRE STUDY}

The Bagust paper is based on a 12 month audit from a single centre in the UK, the Cardiothoracic Centre-Liverpool. The main outcome measured was the potential reduction in risk of repeat revascularisation produced by the use of DES rather than bare metal stents within 12 months of an index procedure. A proportional hazard model for the risk of repeat revascularisation within 12 months was used to identify factors associated with restenosis. In elective patients calcification, angulation $>45^{\circ}$, treatment of restenotic lesions, and triple vessel disease were identified and in non-elective patients, only vessel diameter of $<2 \mathrm{~mm}$ and prior coronary artery bypass grafting. Based on these data the Liverpool group conclude that in elective patients DES are only cost effective (based on the usually quoted UK cost effectiveness threshold of $£ 30000$ per quality of life-year (QUALY) gained) when two or more of the identified factors were present. In non-elective patients DES are only effective if they are placed in vein grafts of $<2 \mathrm{~mm}$ in diameter (that is, both risk factors present). These non-randomised data, from a single centre, suggest that the previous NICE guidelines were incorrect and has potential huge consequences for interventional cardiology in the UK.

Unfortunately, Bagust and colleagues ${ }^{1}$ fail to discuss, in any detail, these findings in the context of multiple other randomised (bare metal stent) studies which have consistently identified the presence of diabetes, small vessels, and longer lesions (the basis of the previous NICE guidelines) as multivariate predictors of target lesion revascularisation (TLR) or target vessel revascularisation (TVR). ${ }^{3-6}$ These factors have also proved positively predictive in DES trials using both sirolimus and paclitaxel. ${ }^{7-9}$

\section{ARE THE CONCLUSIONS VALID?}

After an extensive review of the randomised literature the previous NICE guidelines concluded that DES should be used if the lesion was small $(<3 \mathrm{~mm})$ or long $(>15 \mathrm{~mm})$. It was assumed that this would encompass virtually all diabetics, a group widely recognised to have a major risk of restenosis. ${ }^{10}$ The fact that these factors were not identified in the study by Bagust and colleagues ${ }^{1}$ represents a systematic flaw in these data. The only reasonable conclusions are: (1) the database is inadequate; (2) Liverpool patients are different to the rest of the world; or (3) the patient population is subject to an important selection bias.

Given it is unlikely that Liverpool patients are in any way different, what information are we told about the database? Unfortunately, the answer is very little. Because of the controversial aspects of the paper (the conclusions about risk factors for restenosis fly in the face of the world literature), this is inadequately covered. How were follow up data collected? Is there systematic 30 day, six month, or one year outpatient attendance, patient questionnaire, or telephone interview? My understanding is that none of the above is routinely carried out. In addition, if patients have repeat procedures at another institution, either within or outside the UK, is this recorded by the current database? Again, my understanding is this is not the case. What about the patient population? Is it possible that some systematic bias (widely recognised in registry data) could account for the variance. The absolute need for repeat revascularisation in the Liverpool patients is described as "lower than commonly understood" at $5.6 \%$ in elective patients and $9.0 \%$ in non-elective patients. Understanding this is difficult as the full details of the patient population is not adequately described. No detail is given on vessel size or lesion length. Diabetes is recorded as 13\% which is lower than the $25 \%$ seen in the SIRIUS trial. ${ }^{11}$ Finally, the incidence of left anterior descending coronary artery (LAD) intervention is never stated. It is possible that a systematic bias away

Abbreviations: DES, drug eluting stents; LAD, left anterior descending coronary artery; NICE, National Institute for Health and Clinical Excellence; QUALY, quality of life-year; RAVEL, randomised study with the sirolimus eluting Bx Velocity balloon expandable stent in the treatment of patients with de novo native coronary artery lesions; SIRIUS, sirolimus-eluting balloon expandable stents in the treatment of patients with de novo native coronary artery lesions; TLR, target lesion revascularisation; TVR, target vessel revascularisation 
from diabetes and treatment of LAD lesions by angioplasty with bare metal stents may start to explain the results of this registry.

Unfortunately, the only conclusions that can be drawn from the identified (or indeed unidentified) factors for restenosis in the described population is that either the database is inadequate or the patient population is subject to a major systematic bias. Either of these will mean the conclusions of the study are difficult to transpose into a "real world" UK or international population.

\section{OTHER ISSUES}

There are other aspects of this paper that deserve comment. It is stated that stent trials fail to report "all revascularisation", instead reporting "angiographic restenosis (not all clinically significant), and event rates specific to the lesion or vessel initially revascularised". This is described as "selective reporting" and it is argued that it "exaggerates the apparent benefit attributable to DES". In the SIRIUS trial, " the Cypher stent was associated with a $75.6 \%$ reduction in TLR at 12 months, and a $68.3 \%$ reduction in TVR. The use of TLR certainly does not qualify as "selective reporting" of the trials and is the most sensitive measure of the effect of the device.

The second issue worthy of discussion is described as "protocol driven follow up angiography which overstates both the risk of recurrence and the benefit of using DES". These trials report angiographic outcomes because the follow up angiogram is an important part of the investigation of the safety and efficacy of a DES. In several studies the revascularisation is clinically mandated. The timing relates to the well known and extensively published description of the restenotic process. It allows investigators to check for potential adverse effects of DES on the stented artery and enables the measurement of continuous variables such as late loss which gives a scientific assessment of the biological effect of the drug/polymer combination. ${ }^{12}$ In the 12 month SIRIUS data ${ }^{11}$ the difference in clinically driven TLR event rates between the sirolimus eluting stent and bare metal stent groups in all patients (that is, including those who had protocol mandated angiographic follow up) was $15.1 \%$ at one year. The equivalent measure $(14.0 \%)$ was also reported for the patients who did not have angiographic follow up, indicating that the treatment effect in the absence of the angiogram is virtually the same. Furthermore the proportion of patients whose TLR was based solely on angina symptoms, without consideration of other modalities such as exercise testing, was $96.2 \%$ for sirolimus eluting and $81.0 \%$ for control stent patients. If the 12 month clinically driven TLR rates of $4.9 \%$ for sirolimus eluting and $20.0 \%$ for control stent patients are further adjusted for these respective percentages, this gives 12 month angina driven TLR rates of $4.7 \%$ for sirolimus eluting stents and $16.2 \%$ for control stent patients, a difference of $11.5 \%$ and a $71.0 \%$ "treatment" effect.

Finally, are there other "cost effective" data available in the literature? Two recent publications have demonstrated the cost effectiveness of DES. A Canadian study estimated the cost effectiveness of the Cypher stent over a patient's lifetime by applying the relative risk of repeat revascularisation derived from a meta-analysis of four Cypher trials to absolute risk data from the Alberta province. ${ }^{13}$ This study showed that the Cypher stent was associated with an overall cost effectiveness of $£ 25100$. It was also noted that DES were more cost effective in patients at higher risk of restenosis and, interestingly, showed an incremental cost per QALY gained of $£ 18850$ in diabetic patients. A different approach was taken by van Hout and colleagues, ${ }^{14}$ who conducted a cost effectiveness analysis based on actual resource use in the RAVEL trial using Dutch cost data. The authors acknowledged the potential impact of the follow up angiogram and adjusted the analysis to show the impact of only clinically driven events. At one year, total costs were $£ 113$ higher in the Cypher arm, which the authors described as an "attractive balance between costs and effects for sirolimus-eluting stents".

\section{EVIDENCE BASED PRACTICE}

Cardiology is fortunate in that most important clinical questions have been addressed in properly performed randomised clinical trials. These trials form the basis of evidence based clinical practice and have always been the bedrock of national directives such as those delivered by the NICE committee. Recently, two observational registries have received high profile publications in high quality UK journals and have advocated treatment strategies different to the randomised data and national guidelines. The GRACE registry ${ }^{15}$ recently published in the BMJ suggested that urgent angiography and revascularisation was not necessary, indeed may be harmful, in the setting of unstable coronary syndromes despite the abundance of randomised data suggesting the opposite approach. Now, in this issue of Heart, Bagust and colleagues ${ }^{1}$ suggest DES are only cost effective in $4 \%$ of the population, again despite the vast array of clinical data suggesting most patients would benefit from the deployment of these stents.

I believe that journals publishing such data need to show responsibility and ensure a rigorous pier review process. In addition, I believe, the journal should always consider a balancing view point (as in this case, but I note this did not occur in the $B M J$ ). We all hope that clinicians can critically review the literature as a whole and will give appropriate weight to carefully performed randomised data compared to observational registries. Unless this is the case then certain patient groups risk being inappropriately treated. To suggest that DES could be limited to less than 5\% of patients, ignoring groups with known positively predictive factors, may be putting many patient groups at risk of unnecessary repeat procedures and may not be cost effective for the National Health Service.

\section{REFERENCES}

1 Bagust A, Grayson AD, Palmer ND, et al. Cost effectiveness of drug eluting coronary artery stenting in a UK setting: cost-utility study. Heart 2005;92:68-74.

2 National Institute of Clinical Excellence. Full guidance on the use of coronary artery stents (Technology appraisal guidance no 71); 2003. Available from: www.nice.org.uk.

3 Kastrati A, Schömig A, Elezei S, et al. Predictive factors of restenosis after coronary stent placement. J Am Coll Cardiol 1997;30:1428-36.

4 Kornowski R, Mehran R, Satler L, et al. Procedural results and late outcomes following multivessel coronary stenting. J Am Coll Cardiol 1999;33:420-6.

5 lakovou I, Mintz GS, Dangas G, et al. Optimal final lumen area and predictors of target lesion revascularisation after stent implantation in small coronary arteries. Am J Cardiol 2003;92:1171-6.

6 Singh M, Gersh BJ, McClelland RL, et al. Predictive factors for ischemic target vessel revascularisation in the prevention of restenosis with Tranilast and its outcomes (PRESTO) trial. J Am Coll Cardiol 2005:45:198-203.

7 Moses JW, Leon MB, Popma JJ, et al. Sirolimus-eluting stents versus standard stents in patients with stenosis in a native coronary artery. N Engl J Med 2003;349:1315-23.

8 Stone GW, Ellis SG, Cox DA, et al. One-year clinical results with the slowrelease, polymer-based, paclitaxel-eluting TAXUS stent: the TAXUS-IV trial. Circulation 2004;109:1942-7.

9 Nikolsky E, Kosinski E, Mishkel GJ, et al. Impact of obesity on revascularisation and restenosis rates after bare-metal and drug-eluting stent implantation (from the TAXUS-IV trial). Am J Cardiol 2005;95:709-15

10 Hermiller JB, Raizner A, Cannon L, et al for the TAXUS-IV Investigators. Outcomes with the polymer-based paclitaxel-eluting TAXUS stent in patients with diabetes mellitus: the TAXUS-IV trial. J Am Coll Cardiol 2005; 45: 1 172-9.

11 Holmes DR, Leon MB, Moses MD JW, et al. Analysis of 1-year clinical outcomes in the SIRIUS trial: a randomized trial of a sirolimus-eluting stent 
versus a standard stent in patients at high risk for coronary restenosis. Circulation 2004; 109:634-40.

12 Mauri L, Orav EJ, O'Malley JA. Relationship of late loss in lumen diameter to coronary restenosis in sirolimus-eluting stents. Circulation 2005;111:321-7.

13 Shrive FM, Manns BJ, Galbraith PD, et al. Economic evaluation of sirolimuseluting stents. Can Med Assoc J 2005; 172:345-51.
14 van Hout BA, Serruys PW, Lemos PA, et al. One year cost effectiveness of sirolimus eluting stents compared with bare metal stents in the treatment of single native de novo coronary lesions: an analysis from the RAVEL trial. Heart 2005;91:507-12.

15 Van de WF, Gore JM, Avezum A, et al. Access to catheterisation facilities in patients admitted with acute coronary syndrome: multinational registry study. BMJ 2005;330:441

\section{EDITORIAL}

\section{Heart Review series}

W are pleased to announce a new venture for Heart, the "Heart Review". We are going to commission a series of review articles from recognised authorities in their respective fields, mainly utilising the considerable expertise of our editorial board members. Whereas editorials are generally linked to articles published in the journal and the education series provides a learning template from more established wisdom, linked to the cardiology curriculum for trainees, this new series will bridge the gap between the two. The reviews are designed to give the reader an authoritative assessment of a contemporary subject area with focused, up to date references and will be published online first. We will still accept other reviews of outstanding quality, as before, but hope that this innovation will provide the reader with valuable insights into a wide variety of topical subjects. As ever, we are keen to receive feedback on this or any other aspect of the journal.

\section{IMAGES IN CARDIOLOGY}

\section{Arterial tortuosity syndrome in a newborn}

A newborn was referred to our department for suspected cardiac malformation because of an abnormal elongation of pulmonary arteries detected on the prenatal echocardiography. On postnatal examination, the child presented with downslanting palpebral tissue, overfolded helices, cutis laxa, and joint laxity. Skin fibroblasts, molecular analysis, and lymphocyte chromosome showed no evidence of connective tissue disorder such as EhlersDanlos type IV syndrome or deletion of an elastin gene on chromosome 7. Angiography confirmed the presumptive echocardiographic diagnosis of arterial tortuosity syndrome (ATS), revealing a generalised tortuosity and elongation of all major arteries (panels $\mathrm{A}-\mathrm{C}$; to view video footage visit the Heart website-http://www.heartjnl.com/supplemental).

ATS is a newly defined genetic syndrome in which is found arterial tortuosity associated with hyperextensible skin and hypermobility of joints. While alteration of elastic fibres is suggestive of a connective tissue disorder, little is known about the underlying genetic cause and the clinical evolution of this disorder. The mode of inheritance seems to be autosomal recessive. Recently a locus was identified at chromosome 20q13.

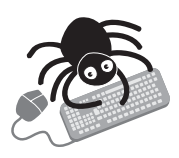

To access video files visit the Heart website-http:// www.heartinl.com/supplemental
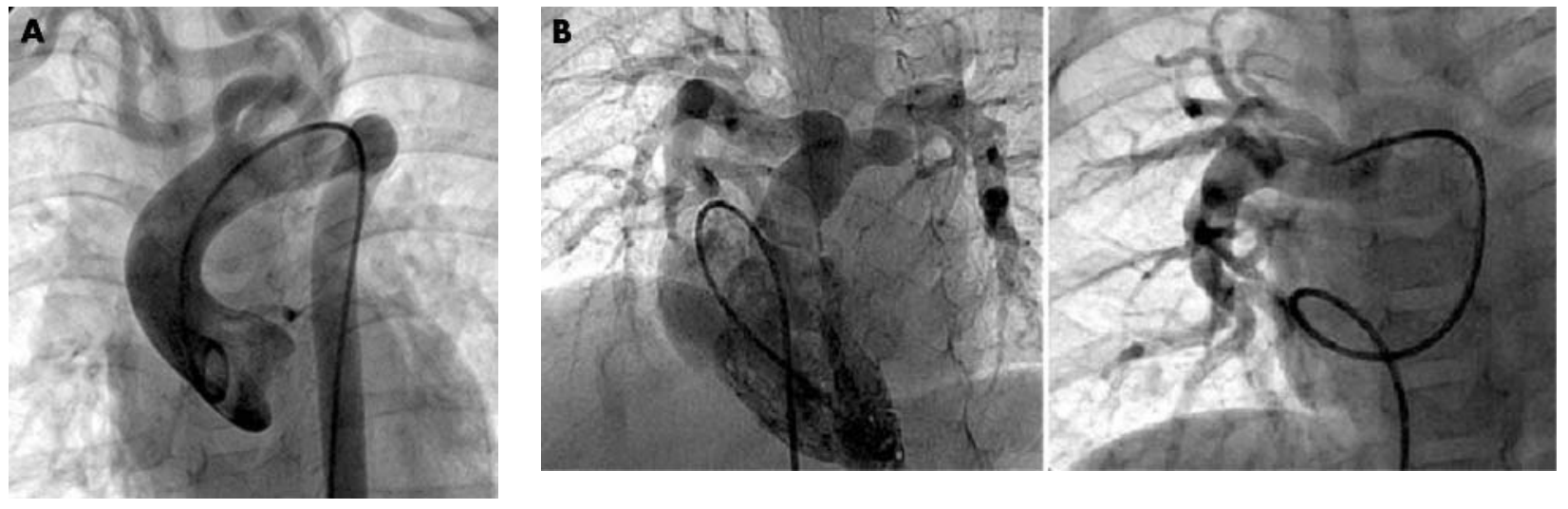